\title{
Late-onset vs nonmendelian early-onset Alzheimer disease
}

\section{A distinction without a difference?}

Christiane Reitz, MD, PhD, Ekaterina Rogaeva, PhD, and Gary W. Beecham, PhD

Neurol Genet 2020;6:e512. doi:10.1212/NXG.0000000000000512

\author{
Correspondence \\ Dr. Reitz \\ cr2101@cumc.columbia.edu
}

\begin{abstract}
There is mounting evidence that only a small fraction of early-onset Alzheimer disease cases (onset $<65$ years) are explained by known mutations. Even multiplex families with early onset often also have late-onset cases, suggesting that the commonly applied categorization of Alzheimer disease into early- and late-onset forms may not reflect distinct underlying etiology. Nevertheless, this categorization continues to govern today's research and the design of clinical trials. The aim of this review is to evaluate this categorization by providing a comprehensive, critical review of reported clinical, neuropathologic, and genomic characteristics of both onsetbased subtypes and explore potential overlap between both categories. The article will lay out the need to comprehensively assess the phenotypic, neuropathologic, and molecular variability in Alzheimer disease and identify factors explaining the observed significant variation in onset age in persons with and without known mutations. The article will critically review ongoing large-scale genomic efforts in Alzheimer disease research (e.g., Alzheimer Disease Sequencing Project, Dominantly Inherited Alzheimer Network, Alzheimer Disease Neuroimaging Initiative) and their shortcomings to disentangle the delineation of unexplained nonmendelian earlyonset from late-onset and mendelian forms of Alzheimer disease. In addition, it will outline specific approaches including epigenetic research through which a comprehensive characterization of this delineation can be achieved.
\end{abstract}

\footnotetext{
From the Taub Institute for Research on Alzheimer's Disease and the Aging Brain (C.R.), Gertrude H. Sergievsky Center (C.R.), Department of Neurology (C.R.), and Department of Epidemiology (C.R.), College of Physicians and Surgeons, Columbia University, New York, NY; Tanz Centre for Research in Neurodegenerative Disease (E.R.), University of Toronto, ON, Canada; and The John P. Hussman Institute for Human Genomics (G.W.B.), University of Miami, FL.

Go to Neurology.org/NG for full disclosures. Funding information is provided at the end of the article.

The Article Processing Charge was funded by the authors.

This is an open access article distributed under the terms of the Creative Commons Attribution-NonCommercial-NoDerivatives License 4.0 (CC BY-NC-ND), which permits downloading and sharing the work provided it is properly cited. The work cannot be changed in any way or used commercially without permission from the journal.
} 


\section{Glossary}

$\boldsymbol{\alpha S y n}=\boldsymbol{\alpha}$-synuclein; AD = Alzheimer disease; ADGC = Alzheimer Disease Genetics Consortium; ADNI $=$ Alzheimer Disease Neuroimaging Initiative; $\mathbf{C N V}=$ copy number variation; $\mathbf{C p G}=$ cytosine-phosphate-guanine; DIAN-OBS = Dominantly Inherited Alzheimer Network observational study; DNAm = DNA methylation; EOAD = early-onset AD; FTD = frontotemporal dementia; GWAS = genome-wide association study; LOAD = late-onset AD; mEOAD = mendelian EOAD; NFL = neurofilament light chain; NFT = neurofibrillary tangle; nmEOD = nonmendelian EOAD; NPC = Neuropathology Core; PiB = Pittsburgh compound B; READR = Resource for Early-onset Alzheimer Disease Research.

Alzheimer disease $(\mathrm{AD})$ is the most common form of dementia accounting for $50 \%-75 \%$ of dementia cases. ${ }^{1}$ To date, an estimated 5.4 million Americans have $\mathrm{AD}$; this number is projected to quadruple by midcentury, largely driven by population aging. By 2050, it is projected that a new case of $\mathrm{AD}$ will develop every 33 seconds on average, resulting in nearly a million new cases each year. ${ }^{1}$ Overall, the incidence of $\mathrm{AD}$ increases exponentially with age, doubling every 5 years after age 65 years, although some individuals, even in multiplex $\mathrm{AD}$ families, successfully age without cognitive decline. ${ }^{2,3}$ Currently, 1 in 9 people aged 65 years and older and 1 in 3 people older than 85 years have the disease. There are no substantially effective therapeutic interventions for $\mathrm{AD}$ available. ${ }^{1}$ Although deaths resulting from heart disease, prostate cancer, and stroke have decreased over the past decade, deaths from $\mathrm{AD}$ have increased by $145 \%$, making $\mathrm{AD}$ the sixth leading cause of mortality in the United States. ${ }^{1}$ Total costs for health care, long-term care, and hospice services for people with dementia are expected to increase from approximately $\$ 290$ billion to date to more than $\$ 1.1$ trillion by the year 2050. Not accounted for in these numbers are the billions of hours of care provided by unpaid caregivers estimated to be equivalent to nearly $\$ 234$ billion annually. ${ }^{1}$

\section{Categorization into early- and late- onset forms of $\mathrm{AD}$}

$\mathrm{AD}$ is commonly categorized as either early onset (EOAD) or late onset (LOAD) based on an age cutoff, typically 65 years. ${ }^{4,5}$ This threshold, however, is arbitrary, and a cutoff of 60 years is also commonly used. Of all patients with $\mathrm{AD}, 5 \%-10 \%$ (corresponding to $220,000-640,000$ Americans) ${ }^{6}$ are attributed to EOAD. Specifically, the $45-64$ years age group has a reported annual incidence of $6.3 / 100,000$ and prevalence of 24.2/ 100,000 , with both rates rising exponentially as individuals approach age 65 years. ${ }^{7,8}$ However, unlike LOAD for which an extensive number of representative data on prevalence and incidence are available, these estimates for EOAD are derived from significantly less data from nationally (and internationally) representative samples, with a paucity of data both within and across ethnic groups. It is possible that the true prevalence and incidence rates for EOAD are higher, in particular for sporadic cases and nonmendelian familial EOAD cases. A substantial portion of those who would meet the diagnostic criteria for $\mathrm{AD}$ are not diagnosed by a physician and so underreported.
In general, there are 2 types of inheritance patterns observed in EOAD: mendelian (mEOAD) and nonmendelian (nmEOAD) patterns. mEOAD forms are typically fully penetrant with an autosomal dominant inheritance pattern, most often caused by mutations in APP, PSEN1, and PSEN2 (see Genetics of mEOAD section). Conversely, nmEOAD is often sporadic or with inconsistent inheritance patterns (i.e., inheritance patterns that are not obviously autosomal dominant, or with highly variable age at onset, including LOAD). Although the etiology and genetic basis of nmEOAD are largely unknown, it is generally considered to be multifactorial, with polygenic effects.

Among the scientific literature, the terminology used to describe EOAD and its subtypes is inconsistent. For many EOAD is synonymous with $\mathrm{mEOAD}$, although $\mathrm{mEOAD}$ accounts only for approximately $10 \%$ of EOAD cases. ${ }^{4,5}$ Some use the terms familial EOAD or autosomal dominant EOAD to refer to $\mathrm{mEOAD}$. Still others use familial EOAD to refer to any EOAD case with a positive family history (whether autosomal dominant) and sporadic EOAD for cases without a family history. This inconsistency in terminology reflects a lack of understanding of the molecular etiology of EOAD, EOAD subtypes, and their delineation from LOAD. Although there is a general consensus that $\mathrm{mEOAD}$ represents a genetic etiology distinct from LOAD, it is less clear if and how nmEOAD is distinct from LOAD. As indicated above, it is generally held that most nmEOAD, both with and without a family history, is predominantly polygenic. Many hypothesize that nmEOAD is simply an extreme phenotype of LOAD that results from an accumulation of variants typically associated with LOAD or the presence of additional genetic or environmental modifying factors lowering onset age (e.g., cerebrovascular disease)., ${ }^{4,5}$ However, there has been little work performed to actually test and verify this hypothesis, posing a critical gap in $\mathrm{AD}$ research and our understanding of $\mathrm{AD}$ etiology. Clarifying to what extend $\mathrm{nmEOAD}$ is clinically and etiologically distinct from mEOAD and LOAD is vital for understanding the molecular mechanisms leading to $\mathrm{AD}$, determining accurate population burden, predicting an individuals' risk, applying appropriate study designs from basic research to clinical trials, and developing personalized and effective targets for prevention and treatment. The purpose of this review is to address this critical gap of knowledge by assessing and summarizing the evidence for differences in etiology between the various presentations of $\mathrm{AD}$ and identifying the void that remains in our understanding of these presentations. 


\section{Clinical characteristics of EOAD and LOAD}

The typical clinical presentation of $\mathrm{AD}$ is characterized by predominant impairment of anterograde episodic memory that, as the disease progresses, is accompanied by dysfunction in additional cognitive domains such as visuospatial, language, and executive function, eventually resulting in global cognitive decline, complete dependency, and death. This typical memory-predominant phenotype is observed in most LOAD cases and a large subset of EOAD cases. ${ }^{9}$ However, roughly $25 \%$ of all subjects with EOAD show an atypical clinical presentation that is characterized by preserved episodic memory but focal cortical symptoms, in particular apraxia, visual dysfunction, aphasia, or executive dysfunction. ${ }^{10-12}$

The clinical characteristics of $\mathrm{mEOAD}$ have been researched through projects such as the Dominantly Inherited Alzheimer Network observational study (DIAN-OBS) and other studies. ${ }^{2,13-17}$ Data from these initiatives suggest that in mEOAD, cognitive symptoms overlap substantially with typical sporadic LOAD symptoms, but usually with a more aggressive disease course with shorter relative survival time. ${ }^{11}$ Noncognitive clinical manifestations affect a subset of participants with mild to moderate mEOAD ${ }^{16}$ The most prevalent nonamnestic cognitive manifestations in participants in the DIAN-OBS cohort are those typical of mild to moderate $\mathrm{AD}$, including visual agnosia (55.1\%), aphasia (57.9\%), and behavioral changes $(61.7 \%)$, whereas the prevalence of noncognitive neurologic manifestations was reported to be lower: myoclonus and spasticity (9.3\%), seizures $(2.8 \%)$, and parkinsonism (11.2\%). Analyses of published data on subjects with $\mathrm{mEOAD}$ yielded a higher prevalence for myoclonus (19.4\%), spasticity (15.0\%), parkinsonism (12.5\%), and seizures $(20.3 \%)$ but a lower prevalence for nonamnestic cognitive manifestations (e.g., visual agnosia [5.6\%], aphasia [23.0\%], and behavioral changes [31.7\%]). ${ }^{16}$ Such discrepancies could be due to differences in disease stage, phenotyping, and/or ascertainment protocols between studies. A recent larger prospective cohort study reported that a significant minority $(16 \%)$ of subjects with $\mathrm{mEOAD}$ had nonamnestic cognitive phenotypes, and about $25 \%$ had atypical neurologic symptoms in addition to the amnestic phenotype. ${ }^{18}$ Analyses of clinical presentation based on genetic background suggest that compared with APP carriers, PSEN1 carriers are more likely to exhibit additional neurologic features such as myoclonus, corticobulbar deficits, aphasia, and spasticity but less often ischemic stroke and hemorrhage. ${ }^{16,18}$ Also, mutation location within the gene could affect the clinical phenotype. ${ }^{18}$ For instance, most of the APP substitutions at the $A \beta$ sequence lead to $\mathrm{mEOAD}$, whereas the Ala673Thr APP protects against of $\mathrm{AD}{ }^{19}$

There is a lack of studies assessing the clinical characteristics of $n m E O A D$ as a specific disease entity separate from mEOAD and LOAD. The reported studies were heterogeneous in their design, applied a variety of diagnostic criteria, often did not consider biomarkers, and typically assessed all types of dementia without excluding mEOAD, hindering informed conclusions to be drawn about this form of $\mathrm{AD} .^{7}$ Like mEOAD and LOAD, nmEOAD is typically memory predominant, with nonamnestic symptoms in a subset of the patients. However, data beyond this are limited. It is not clear whether the neuropsychological profile within the memory domain differs from that of $\mathrm{mEOAD}$ and LOAD. In addition, the true prevalence of atypical presentations within the nmEOAD category remains unclear, as does the true onset age distribution of both sporadic and familial nmEOAD cases.

\section{Neuropathologic distinction between EOAD and LOAD}

$\mathrm{AD}$ is neuropathologically characterized by extracellular accumulation of diffuse and neuritic amyloid plaques composed of abnormally folded $A \beta 40$ and $A \beta 42$ generated by cleavage of APP, and intraneuronal accumulation of neurofibrillary tangles (NFTs) composed of hyperphosphorylated tau protein (p-tau). These pathologic features are often accompanied by neuropil threads, dystrophic neurites, associated astrogliosis, microglial activation, and cerebral amyloid angiopathy. ${ }^{20}$ Furthermore, $50 \%$ of patients with $\mathrm{AD}$ exhibit concurrent $\alpha$-synuclein ( $\alpha$ Syn) pathology. ${ }^{21,22}$ Although amyloid deposition does not always follow a fixed pattern of progression, it usually develops in the isocortex and affects subcortical structures later in the disease process. In contrast, tau pathology begins typically in the allocortex of the medial temporal lobe involving the entorhinal cortex and hippocampus. ${ }^{23}$ In general, $A \beta$ pathology reaches a plateau in the early symptomatic phase of the disease, ${ }^{24}$ whereas NFT pathology correlates more closely with clinical features, severity, and progression of $\mathrm{AD}^{20}$ There is, however, a certain degree of disconnect between these neuropathologic changes and clinical symptoms; individuals with a high burden of $\mathrm{AD}$ pathology are sometimes without clinical symptoms, and individuals with a significant degree of cognitive impairment sometimes exhibit limited $\mathrm{AD}$ pathology. ${ }^{23}$

To what extent the neuropathology of nmEOAD, mEOAD, and LOAD overlap remains unclear. Compared with older people who often show multiple comorbid pathologies other than $\mathrm{AD}$ (e.g., Lewy bodies, TDP-43, or vascular pathology), $\mathrm{AD}$ at a younger age tends to be associated with more pure $\mathrm{AD}$ pathology. ${ }^{25-27}$ In line with this notion, a number of studies using MRI and PET scans have reported a larger burden and more widespread pathology with neocortical and subcortical involvement in younger cases, see the section entitled "Brain imaging and biofluid biomarkers" below. ${ }^{10,28-32}$ The brain pathology of some variant $\mathrm{AD}$ cases is characterized by diffuse cotton wool $A \beta$-plaques without neuritic pathology or neuroinflammation. ${ }^{33}$ Most of the 
variant $\mathrm{AD}$ cases are PSEN1 carriers often affected by spastic paraparesis. ${ }^{34}$ However, the cotton wool plaques were also reported in a few $L O A D$ cases, suggesting that this brain pathology is not entirely specific for mEOAD. ${ }^{35}$ Cotton wool $\mathrm{A} \beta$-plaques might result from an inhibited nucleation stage of fibrillogenesis in the presence of a particular genetic background. Furthermore, there is evidence that different fibril structures of $A \beta$ peptides might underlie different courses and time frames of disease progression. ${ }^{36}$

A critical shortcoming of most neuropathology studies is the lack of genetic information to distinguish between carriers and noncarriers of causal/risk variants and patterns of inheritance (e.g., mEOAD vs nmEOAD). Recognizing the urgency to better define the pathologic distinction between $\mathrm{MEOAD}$ and LOAD, the Alzheimer Disease Neuroimaging Initiative (ADNI)/DIAN Neuropathology Core (NPC) has recently implemented a uniform assessment protocol to evaluate the neuropathologic overlap between these 2 forms. ${ }^{37}$ As part of this protocol, multiple histologic stains are performed on all brains that include hematoxylin and eosin, a modified Bielschowsky silver impregnation, and immunohistochemistry using the following primary antibodies: phosphorylated tau (PHF1), $\beta$-amyloid (10D5; Eli Lilly, Indianapolis, IN), phosphorylated aSyn (Cell Applications, San Diego, CA), and phosphorylated TDP-43 (Cosmo Bio, Carlsbad, CA). NACC operational criteria for the classification of $\mathrm{AD}$ and other pathologies are applied, which include diagnosis of $\mathrm{AD}$ by several sets of criteria (Khachaturian, CERAD, NIA-Reagan, and the NIA-AA $\left.{ }^{38-40}\right)$. However, there is no coordinated research effort to evaluate the overlap with nmEOAD.

\section{Brain imaging and biofluid biomarkers}

Data on mEOAD from the DIAN study suggest that compared with noncarriers, carriers of causal mutations show reduced cortical glucose metabolism and cortical thinning in the medial and lateral parietal lobes 5-10 years before the estimated age at onset and widely distributed elevated Pittsburgh compound $\mathrm{B}(\mathrm{PiB})$ levels in nearly every cortical region approximately 15 years before estimated onset. ${ }^{41}$ Subcortical regions seem to show a disparate pattern, with all subcortical gray matter regions exhibiting elevated $\mathrm{PiB}$ uptake, but reduced glucose metabolism being apparent only in the hippocampus. ${ }^{41}$ Atrophy in mEOAD is observed early, both in areas commonly associated with sporadic LOAD and additional areas including the putamen and thalamus, which are associated with early amyloid deposition in mEOAD. ${ }^{42}$ Tau tracer binding in the neocortex is also increased in symptomatic mutation carriers compared with sporadic $\mathrm{LOAD}^{43}$; however, it remains to be clarified which component of tauopathy (e.g., NFT, neuritic plaques, or neuropil threads) is reflected by this increased PET-tau signal.

Longitudinal analyses in the DIAN study ${ }^{17}$ aim to pinpoint onset, sequence, and rate of progression of biomarker and clinical measures across the spectrum of mEOAD. The results from this study suggest that $\beta$-amyloid measured in CSF change first, decades before estimated symptom onset, followed by declines in measures of cortical metabolism approximately 7-10 years later, then cognition and hippocampal atrophy (approximately 2 decades after changes in $\beta$-amyloid). CSF p-tau 181 showed a marked decline near symptom onset; this is in contrast with previous observations from cross-sectional analyses, which suggested a continued elevation of CSF p-tau 181 . There is also evidence that CSF $\alpha$ Syn levels are linked to onset of cognitive symptoms in both sporadic $\mathrm{LOAD}$ and $\mathrm{mEOAD}^{22}$ and that CSF progranulin, encoded by $G R N$, increases over the course of disease and is associated with sTREM2, neurodegeneration, and cognitive decline. $^{44}$

Studies in subjects with $\mathrm{nmEOAD}$ without $\mathrm{AD}$ family history have also shown more atrophy, ${ }^{45}$ more prominent neocortical glucose hypometabolism, ${ }^{46}$ and increased tau PET uptake ${ }^{47,48}$ relative to LOAD. This is in line with findings at brain autopsy, indicating that neurodegeneration and tauopathy are more aggressive at a younger onset, but the number of studies is limited. Even more limited is the availability of longitudinal imaging and biomarker data sets of nmEOAD multiplex families and direct comparison with the mendelian form of EOAD. Analyses of the ADNI data set (unrelated $\geq 65$-yearold subjects) applying linear mixed-effects models also demonstrate that LOAD defined by MRI and biofluid-based biomarkers proceeds more aggressively among younger than older elderly individuals. ${ }^{49}$ Specifically, this study suggests that brain atrophy rates in $\mathrm{AD}$ (and mild cognitive impairment) are stronger with younger baseline age. Conversely, atrophy rates for clinically normal older individuals remain constant or exhibit a slight increase with age (slopes [\% atrophy/year $\left.{ }^{2}\right]$ ranged from -0.04 to 0.006 and was only significant for the hippocampus [slope: $-0.04 ; p=0.004]){ }^{49}$ Similarly, younger individuals with $\mathrm{AD}$ or mild cognitive impairment exhibit a greater CSF biomarker burden, whereas among healthy controls, biomarker burden increases with age. In addition, $\mathrm{AD}$ cases show reduced rates of cognitive decline with increasing baseline age. ${ }^{49}$ Together, these data support the notion of a disease continuum across the age range in LOAD, up to a blurring of the distinction between $A D$ and healthy controls older than 85 years. ${ }^{49}$ However, whether this continuum truly extends to the younger age range and $\mathrm{nmEOAD}$ remains to be assessed.

Efforts to develop reliable, sensitive, and specific biomarkers of blood-based biomarkers for $\mathrm{AD}$ (e.g., plasma or serum) have been hampered by various challenges, in particular much lower amounts of molecules of interest in the bloodstream compared with the CSF and high levels of other proteins from peripheral organs in the blood and presence of proteases that degrade brain proteins. However, in recent years, there have been significant advances in the development of highly sensitive assays to assess plasma levels of molecules that could serve as biomarkers of $\mathrm{AD}$ and other types of 
neurodegeneration for diagnosis, prognosis, and disease progression monitoring. ${ }^{50,51}$ Various studies have shown that the ratio of plasma $A \beta 42 / 40$ measured by these ultrasensitive assays such as the single-molecule array method provides a sensitive and reliable measure of amyloid status that predicts future conversion to positive amyloid PET independent of the time of day and correlates with CSF $A \beta 42 / 40$ in both mEOAD and LOAD. ${ }^{52-55}$ In addition, neurofilament light chain (NFL), an intraneuronal component of the axonal cytoskeleton reflecting neuronal damage and degeneration, increases in the CSF in early stages of the disease. Also, NFL increases over time as cognition declines and atrophy and white matter changes increase. NFL has diagnostic accuracy for $\mathrm{AD}$ dementia similar to that of CSF biomarkers in subjects with both forms of disease, ${ }^{56,57}$ and studies in individuals with mEOAD, including the DIAN study, suggest that plasma NFL increases several years before symptom onset, indicating its utility as a screening tool in this type of $\mathrm{AD} .{ }^{58-60}$ However, there is a lack of data specifically assessing NFL association in nmEOAD. Current data are less supportive of the use of plasma tau as a useful biomarker for $\mathrm{AD}$, although $\mathrm{p}$-tau $\mathrm{ta}_{181}$ levels are elevated in $\mathrm{AD}$ dementia and show associations with both $A \beta$ and tau PET, suggesting greater specificity for $A D$ pathology than other tau species. ${ }^{\mathrm{e}-1}$

\section{Genetics of mEOAD}

\section{Heritability}

Although LOAD is a complex disorder with a strong genetic component (heritability of 70\%-80\%), ${ }^{4, e-2}$ EOAD in general has an even stronger heritability estimated between $92 \%$ and $100 \%{ }^{4}$ This is in line with the observation that up to $60 \%$ of patients with EOAD have at least 1 affected first-degree relative. e-3,e-4 $^{-\mathrm{s}}$

\section{Genetics of mEOAD}

Known mEOAD results from 1 copy of a mutant allele in APP, ${ }^{\mathrm{e}-5}$ PSEN1, ${ }^{\mathrm{e}-6}$ or PSEN2 $2^{\mathrm{e}-7, \mathrm{e}-8}$; in total, $\sim 330$ mutations in these genes have been reported (alzforum.org/alzgene), accounting for $10 \%-15 \%, 30 \%-70 \%$, and $<5 \%$ of $\mathrm{mEOAD}$ cases, respectively. ${ }^{\mathrm{e}-9}$ However, overall variation in these genes explains only $10 \%-15 \%$ of all patients with familial EOAD. ${ }^{\mathrm{e}-4}$ Mutations in APP, PSEN1, and PSEN2 affect the amyloidogenic pathway leading to increased generation/ aggregation of $A \beta .^{e-10, e-11} A P P$ undergoes cleavage by $\beta$ - and subsequently $\gamma$-secretase generating A $\beta$ peptides; and PSEN1 or PSEN2 are components of the $\gamma$-secretase complex. ${ }^{\text {e-12 }}$ Known pathogenic APP missense substitutions are clustered at the $A \beta$ sequence and linked to higher $A \beta$ production or aggregation, similar to $A P P$ duplications. ${ }^{\mathrm{e}-13}$ In contrast, the protective Icelandic APP substitution (Ala673Thr) decreases A $\beta$ levels by $40 \% .{ }^{19}$ Notably, a mutation at the same codon (Ala673Val) causes $\mathrm{AD}$, but only in a recessive mode. ${ }^{\mathrm{e}-14}$ The brain pathology of APP carriers is often accompanied by a significant level of cerebral amyloid angiopathy, which can lead to cerebral hemorrhage and stroke in carriers of $A P P$ duplications ${ }^{\mathrm{e}-15, \mathrm{e}-16}$ or some substitutions (e.g., Glu693 $\mathrm{Gln}^{\mathrm{e}-5}$ and Glu693 $\left.\mathrm{Lys}^{\mathrm{e}}{ }^{-17}\right)$. However, some of the APP mutations (e.g., Glu693 $\mathrm{Gly}^{\mathrm{e}-18}$ ) were reported in patients without the severe amyloid angiopathy.

In contrast to APP, mutations in PSEN1 or PSEN2 are broadly distributed throughout the gene (alzforum.org/alzgene). Most of them are missense variants with a few in-frame deletions/insertions. ${ }^{33,-\mathrm{e}-19, \mathrm{e}-20}$ Despite considerable sequence, structural, and functional similarities, many more pathogenic mutations were reported in PSEN1 $(\mathrm{n}=263)$ than PSEN2 ( $\mathrm{n}$ =12). PSEN1-related disease is also more severe with onset as early as the third decade (e.g., in Ser170Phe carriers), ${ }^{\mathrm{e}-21}$ whereas some PSEN2 mutations cause LOAD (e.g., Val148Ile). ${ }^{\mathrm{e}-22}$ This discrepancy might be explained by the fact that brain expression of PSEN2 is up to 10 times lower than PSEN1 (FANTOM5 data set; proteinatlas.org/).

Phenotypic variability in PSEN1 patients includes frontotemporal dementia (FTD), Pick-type tauopathy, ${ }^{\mathrm{e}-23, \mathrm{e}-24}$ primary progressive aphasia, spinocerebellar ataxia, ${ }^{\mathrm{e}-25, \mathrm{e}-26}$ Lewy body dementia, ${ }^{\mathrm{e}-27}$ and variant $\mathrm{AD}$ accompanied by spastic paraparesis. ${ }^{34}$ Even within a family, phenotypes of PSEN1 carriers can appear as variant $\mathrm{AD}$, pure $\mathrm{EOAD}$, or pure spastic paraparesis, ${ }^{\mathrm{e}-28}$ suggesting the action of genetic modifier(s).

\section{Genes modifying age at onset in $\mathrm{MEOAD}$}

In general, age at onset of $\mathrm{AD}$ is a substantially heritable trait; however, with increasing age at onset, the heritability of $\mathrm{AD}$ declines, implying an increased environmental contribution to $\mathrm{AD}$ at older ages. ${ }^{4}$ Even within families, carriers of the same $A P P, P S E N 1$, or PSEN2 variant can present with significant variability in age at onset and disease course. A meta-analysis of $387 \mathrm{mEOAD}$ pedigrees showed that the observed variance could only be partially explained by family history, the mutated genes, and the mutation type. ${ }^{\mathrm{e}-29}$

The APOE $\varepsilon 4$ allele, the major genetic risk factor identified for $\mathrm{LOAD}$, decreases age at onset of $\mathrm{AD}$ in a dose-dependent manner. ${ }^{\mathrm{e}-30}$ In contrast to fully penetrant $\mathrm{mEOAD}$ mutations, the $A P O E \varepsilon 4$ allele is neither necessary nor sufficient to cause $\mathrm{AD}$. However, a more recent study suggested that the effect of $A P O E$ on $\mathrm{AD}$ risk is consistent with semidominant inheritance of a moderately penetrant gene. ${ }^{\mathrm{e}-31}$ Furthermore, several studies showed that $A P O E$ has an age at onset-modifying effect in carriers of some causal mutations (e.g., APP Val717lle, ${ }^{\mathrm{e}-32}$ PSEN1 Glu280Ala, ${ }^{\mathrm{e}-33, \mathrm{e}-34}$ PSEN1 Glu318Gly, ${ }^{\mathrm{e}-35}$ and PSEN2 Asn141Ile $\left.{ }^{\mathrm{e}-36}\right)$.

However, the wide range of onset age in carriers of the PSEN1 Glu206Ala mutation (found in $42 \%$ of EOAD families of Caribbean Hispanic origin ${ }^{\mathrm{e}-37}$ ) is not explained by the APOE $\varepsilon 4$ allele $^{\mathrm{e}-29}$ nor any antecedent environmental, health-related, or social factors. ${ }^{\mathrm{e}-38}$ Investigation of Glu206Ala carriers and other 
common founder mutations (e.g., PSEN1 Ala431Glu in Mexicans) can facilitate the search of novel age at onset modifiers. ${ }^{\text {e- }}$ 39,e-40 For instance, genome-wide linkage analyses of Volga German families segregating the PSEN2 Asn141Ile mutation identified 3 genetic loci (1q23.3, 17p13.2, and 7q33) ${ }^{\mathrm{e}-41}$ potentially harboring onset-modifying genes. Whole-genome sequencing analyses of a large Colombian kindred with the PSEN1 Glu280Ala mutation identified a protective haplotype spanning several cytokine genes on chromosome 17 that delayed $\mathrm{AD}$ onset by approximately 10 years. ${ }^{\mathrm{e}-42}$ Intriguingly, a separate study in this kindred identified a carrier of the Glu280Ala variant who developed mild cognitive impairment only in the seventh decade, which is $\sim 30$ years later than the median age at onset in the kindred. ${ }^{\mathrm{e}-43}$ The patient had very high brain amyloid, but limited neurodegeneration and tau pathology. Whole-exome sequencing identified a homozygous Arg136Ser mutation in APOE that was previously associated with hyperlipoproteinemia type III, suggesting that this rare $A P O E$ variant is protective and in a homozygous state may reduce tau pathology and neurodegeneration. ${ }^{\mathrm{e}-43}$

\section{Genetics of LOAD}

Over the past decade, large-scale genome-wide association study (GWAS) and whole-genome/exome sequencing have identified over 30 genome-wide significant common variant signals in addition to $A P O E$ that influence risk for LOAD. ${ }^{\mathrm{e}-44-\mathrm{e}-51}$ These variants mostly point to specific pathways (e.g., lipid metabolism, endocytosis/intracellular trafficking, inflammation, immune response, synaptic function, and transcription) indicating a major role for these pathways in the development of LOAD. Although risk variants in genes involved in tau and APP metabolism (PSEN1, PSEN2, APP, ADAM10, ADAMTS1, MAPT), ${ }^{\mathrm{e}-52-\mathrm{e}-54}$ as well as in genes of other dementing disorders (e.g., GRN, ARSA, CSF1R) were also implicated. ${ }^{\mathrm{e}-55}$ Taken together with the fact that some families carrying APP, PSEN1, or PSEN2 mutations present with $\mathrm{LOAD}^{\mathrm{e}-52}$ strongly corroborates the notion that these pathways are not restricted to $\mathrm{mEOAD}$ and that there is etiologic overlap between the forms commonly classified as EOAD and LOAD.

In addition, some of the known LOAD loci might be age at onset modifiers in EOAD. For instance, analysis of the ABCA7 coding sequence showed a 5-fold enrichment of premature termination codon mutations in patients with EOAD $(n=928)$ vs controls $(\mathrm{n}=980)$. Of 17 observed mutations, 10 were novel and only observed in patients. ${ }^{\text {e-56 }}$ Furthermore, although common variants in SORL1 were first linked to $\mathrm{LOAD}$ with an odds ratio of $\sim 1.2$, e-57,e-58 $^{\text {rare }}$ truncating SORL1 mutations with odds ratios up to 12 are seen in EOAD cases and might need to be considered in clinical practice in addition to PSEN1, PSEN2, and APP..$^{\mathrm{e}-59}$ Overall, the risk variants identified to date account for $\sim 31 \%$ of the genetic variance of $\mathrm{LOAD},{ }^{\mathrm{e}-44}$ leaving the majority of genetic risk as yet uncharacterized.

\section{Genetics of nmEOAD}

Considering that (1) EOAD is almost entirely genetically determined $^{4}$; (2) 35\%-60\% of patients with EOAD have at least 1 affected first-degree relative $\mathrm{e}^{\mathrm{e}-3, \mathrm{e}-4, \mathrm{e}-60}$; and (3) known causal mutations account for less than $10 \%$ of EOAD cases, it is clear that there are unidentified genetic variation(s) with a substantial effect on nmEOAD risk and/or age at onset. The fact that the concordance between the parent and the offspring in nmEOAD cases is lower than $50 \%$ but higher than $2.5 \%{ }^{4}$ indicates that this form of EOAD rather represents a complex disease with the genetic component made up of a significant number of both rare and common variants. In addition, part of the missing heritability of nmEOAD can be hidden within poorly investigated mutation types, such as copy number variations (CNVs). In general, only a limited number of $\mathrm{AD}$ studies have investigated the role of CNVs and other structural variants. $^{\mathrm{e}-61}$ An $18-\mathrm{kb}$ insertion in CR1 (responsible for the CR1-S isoform with an allelic frequency of $15 \%$ resulting in an extra set of $\mathrm{C} 3 \mathrm{~b} / \mathrm{C} 4 \mathrm{~b}$ binding sites) increases $\mathrm{AD}$ risk 2-fold and explains the GWAS signals at the CR1 locus. ${ }^{\mathrm{e}-62} \mathrm{~A}$ 44-base pair frameshift deletion in $A B C A 7$ increases $\mathrm{AD}$ risk in African Americans and $\mathrm{Ca}$ ribbean Hispanics. ${ }^{\mathrm{e}-49}$

A study of 261 nonmendelian families with at least 1 EOAD case revealed 5 deletions and 5 duplications that segregated with dementia, ${ }^{\mathrm{e}-63}$ with $2 \mathrm{CNV}$ s encompassing FTD genes (deletion of CHMP2B and duplication of $M A P T$ ). A recent analysis of Caribbean Hispanic families multiplex for EOAD not explained by APP, PSEN1, or PSEN2 mutations, ${ }^{\mathrm{e}-64}$ identified 8 regions previously associated with the late-onset form, and several novel linkage regions previously not reported in LOAD subjects (2p15, 5q14.1, $11 \mathrm{p} 15.1,13 \mathrm{q} 21.22,13 \mathrm{q} 33.1,16 \mathrm{p} 12.1,20 \mathrm{p} 12.1$, and $20 q 11.21)$. This is in line with the notion that the genetic architectures of nmEOAD and LOAD overlap partially, but not fully.

\section{Role of epigenetic changes}

The role of epigenetic modifiers in $\mathrm{AD}$ is largely unknown; however, both environmental and genetic age at onset modifiers could act through epigenetic changes, such as DNA methylation (DNAm) at specific cytosine-phosphate-guanine ( $\mathrm{CpG}$ ) sites, one of the key epigenetic modifications. The Exome Aggregation Consortium revealed that $\mathrm{CpGs}$ are the most highly mutable sites in the human genome, likely because methyl-C can spontaneously deaminate to $\mathrm{T}$, leading to $\mathrm{C}>\mathrm{T}$ or $\mathrm{G}>\mathrm{A}$ transitions on sense or antisense DNA strands. ${ }^{\mathrm{e}-65}$ As a result, the frequency of CpGs in the genome is $25 \%$ less than expected and $35 \%$ of all coding mutations occur at $\mathrm{CpG}$ sites. The fact that DNAm levels are under strong genetic control is evident by very similar genome-wide DNAm profiles in identical twins or triplets vs fraternal 
siblings. ${ }^{\mathrm{e}-66, \mathrm{e}-67}$ Hence, studies of crosstalk between genetic and epigenetic markers are of significant value. For instance, the analysis of genetic variations controlling the gain or loss of $\mathrm{CpG}$ sites could reveal the functional disease-related $\mathrm{CpG}$ single nucleotide polymorphisms responsible for DNAm changes. Such a study design reduces the likelihood of falsenegative results due to excessive correction for multiple testing used in classic GWAS. ${ }^{\mathrm{e}-68}$

Aging, the strongest risk factor for $\mathrm{AD}$, is closely linked to DNAm. It is clear that aging processes are not sufficiently represented by chronological aging. In contrast to the steady pace of chronological age, the pace of biological age varies among individuals and may be linked with different aspects of aging via age-related CpGs. In the genomic research field, both the number of different DNAm clocks (based on specific GpG sets) and the methodological approaches to assess these clocks are rising due to their potential in predicting health span. $^{\mathrm{e}-69}$ It would be important to establish the age-related expression pattern of genes corresponding to clock-building CpGs. The CpGs of the reported DNAm clocks are mapped to 1,633 different genes, including 106 of that are genetically associated with neurodegenerative diseases, as well as 32 genes involved in the amyloid biological network (e.g., BACE1 and PSEN1). e $69^{\text {am }}$

The Horvath's DNAm clock outperforms others based on its multitissue applicability and may reflect biological aging. ${ }^{\text {e-70 }}$ It is an accurate age predictor across different tissues $(r=0.96)$, including blood and brain, and is based on cumulative assessment $\sim 350 \mathrm{CpGs}$ (available on genome-wide DNAm arrays), which are hyper- or hypomethylated with age and mainly mapped to genes involved in development, cell death, and survival. ${ }^{\mathrm{e}-70}$

DNAm-age acceleration (DNAm-age minus chronological age) has been found to be significantly associated with several neurodegenerative diseases including Parkinson disease, ${ }^{\mathrm{e}-71}$ Huntington disease, ${ }^{\mathrm{e}-72}$ and amyotrophic lateral sclerosis with or without symptoms of FTD. ${ }^{\text {e-73,e-74 }}$ The limited data on DNAm-age in $A D$ suggest that DNAm-age acceleration is a significant predictor of dementia ${ }^{\mathrm{e}-75}$ and correlates with degree of amyloid pathology in $\mathrm{AD} .^{\mathrm{e}-76}$ We recently investigated a unique Ashkenazi Jewish family with monozygotic triplets affected by $\mathrm{LOAD}$, whereas one of their offspring developed EOAD at age 50 years. Notably, DNAm-age of the triplets was 6-10 years younger than chronological age, but it was 9 years older in the offspring with EOAD, suggesting accelerated aging. ${ }^{\text {e- } 66}$ It is not clear whether DNAm-age reacts to aging or causes aging; however, the longitudinal study of identical twins revealed that the asymptomatic twins had aged slower than the affected twins, supporting the latter notion. ${ }^{\mathrm{e}-67}$ Comprehensive characterization of the association of DNAmage acceleration with $\mathrm{AD}$ onset can provide critical information on the distinction between $\mathrm{nmEOAD}, \mathrm{mEOAD}$, and LOAD.

\section{What is lacking in our understanding of the clinical, neuropathologic, and genetic delineation of nmEOAD from MEOAD and LOAD?}

There remain critical gaps that need to be addressed to disentangle the etiologic and clinical delineations between $\mathrm{nmEOAD}, \mathrm{mEOAD}$, and LOAD. Foremostly, there has been a critical lack of longitudinal well-phenotyped data sets of nmEOAD cases and multiplex families, with standardized definition and methodology, in different disease stages, and of different ancestries with clinical, biofluid, neuropathologic, and genomic information. Although some existing EOAD cohorts might harbor nmEOAD cases, emphasis has mostly been put on individuals screening positive for genetic variants. For many data sets, it remains unclear if and how many subjects without known mutations there are and what their clinical biomarker and neuropathologic expression is. Studies specifically sampling nmEOAD cases would allow comprehensive examination of population risk across ethnic groups, longitudinal changes in biomarkers/neuropathology over the disease course, prevalence of atypical nonamnestic and neurologic manifestations, and tease apart clinical, neuropathologic, and genetic overlap with $\mathrm{mEOAD}$ and LOAD, and among sporadic and familial forms.

Second, pinpointing the significant proportion of missing heritability will require large sets of well-phenotyped familybased and case-control studies of nmEOAD cases with suffcient statistical power. Investigation of all types of genetic variation including structural, synonymous, and rare variations, examination of all possible inheritance modes, and comprehensive study of epigenetic changes is needed. Integration of sequencing with data derived from other -omics approaches (e.g., transcriptomics, epigenomics, proteomics, and metabolomics) coupled with advanced bioinformatics will be critical to pinpoint the functional allelic effectors of identified loci, define and understand the cellular expression of disease, characterize determinants and mechanisms of disease progression, and clarify the distinction of disease subtypes.

In-depth genetic studies in additional $\mathrm{mEOAD}$ and $\mathrm{nmEOAD}$ kindreds displaying a marked discrepancy in age at onset between family members will also be highly valuable to disentangle the etiology of differences in age at onset. It has become clear that the location of pathogenic variants within causal genes can affect pathophysiology and age at onset, as has been established for variation in PSEN1 pre- and postcodon $200 .{ }^{16, e-77}$ However, there are limited data of differential effects of variant location within any other $\mathrm{AD}$ genes on age at onset and phenotypic variability, incomplete understanding of the biochemical features of the $\mathrm{AD}$-associated gene domains, and a lack of knowledge on local genetic ancestry at these loci. As has been recently demonstrated for the 
APOE locus, examination of local ancestry can identify novel ancestry-specific disease loci and help disentangle the factors underlying observed differential genome-phenome associations including variability in age at onset. ${ }^{\mathrm{e}-78}$

For both mEOAD and nmEOAD, there is a lack of data comprehensively assessing the effect of $A P O E$ and other established $\mathrm{AD}$ variants on age at onset and clinical variability in mutation carriers, and a lack of data incorporating genomic information and neuropathologic features, changes in biomarker sequence and progression of symptoms. Assessing neuropathologic, biofluid, and brain imaging measures across the disease course informed by genetic background is critical to disentangle the clinical delineation of $\mathrm{AD}$ subtypes associated with specific variants. As described above, the ADNI/ DIAN NPC has implemented a uniform assessment protocol to evaluate the neuropathologic overlap between $\mathrm{mEOAD}$ and LOAD. ${ }^{37}$ Similar collections of nmEOAD brain tissue with standardized neuropathologic assessment are needed to comprehensively delineate neuropathologic overlap of both these forms with sporadic and familial nmEOAD informed by genetic background, acknowledging that-given that pathology is confounded with disease onset, progress, and age at death-differentiation of mEOAD, nmEOAD, and LOAD based on neuropathologic profile is inherently limited.

\section{Conclusions}

Facilitated by substantial advances in genomic technologies and bioinformatics, the past decade has brought significant breakthroughs in our understanding of the genetic etiology and molecular mechanisms underlying $\mathrm{AD}$. In addition to the known EOAD genes and $A P O E$, we have now identified over 30 loci that modify disease susceptibility. These loci robustly implicate specific molecular pathways (e.g., immune response, lipid metabolism, and endocytosis) and have advanced our understanding of the disease mechanisms at play. However, significantly lacking is the understanding of genetic, clinical, and neuropathologic delineation of $\mathrm{nmEOAD}$ from $\mathrm{mEOAD}$ and LOAD. In-depth characterization of the distinction between these forms is critical to fully understand the etiology of $\mathrm{AD}$, risk factors, and clinical determinants of age at onset, inform clinical trials aiming to reposition existing drugs, and develop more effective novel preventive and therapeutic measures advancing the field toward our vision of personalized medicine.

It is possible to fill the research gaps in our understanding of $\mathrm{nmEOAD}$. Although nmEOAD is less common than LOAD, collection and assessment of participants with $\mathrm{nmEOAD}$ is quite feasible. Given the severity of the disease, patients are often enthusiastic to participate in clinical research studies. Such participants present at Alzheimer Disease Centers and other memory clinics with regularity, although follow-up and participation in biomarker and genetic studies are inconsistent. Indeed, there are now novel ongoing efforts to address these issues. Two such studies include efforts out of the Alzheimer Disease Genetics Consortium (ADGC) and the Resource for Early-onset Alzheimer Disease Research (READR). The ADGC and READR have novel wholegenome sequencing studies of well-phenotyped EOAD participants lacking known mutation (ADGC focusing on singleton/sporadic and READR focusing on families). It is expected that these studies will identify novel variants and molecular pathways associated with nmEOAD in different ethnic groups and help delineate the etiology of this form of $\mathrm{AD}$ from mEOAD and LOAD genetically and clinically.

\section{Study funding}

This work was in part supported by NIH grants RF1AG054080 (C.R., G.W.B., E.R.), R01AG064614 (C.R., G.W.B.), U01AG052410 (C.R.), P50AG008702 (C.R.), U01AG052410 (G.W.B.), and RF1AG054074 (G.W.B.). E. Rogaeva was further supported by the Canadian Consortium on Neurodegeneration in Aging.

\section{Disclosure}

The authors report no disclosures relevant to the manuscript. Go to Neurology.org/NG for full disclosures.

\section{Publication history}

Received by Neurology: Genetics April 9, 2020. Accepted in final form August 7, 2020.

Appendix Authors

\begin{tabular}{|c|c|c|}
\hline Name & Location & Contribution \\
\hline $\begin{array}{l}\text { Christiane } \\
\text { Reitz, MD, } \\
\text { PhD }\end{array}$ & $\begin{array}{l}\text { Columbia } \\
\text { University, New } \\
\text { York }\end{array}$ & $\begin{array}{l}\text { Drafting/revising the manuscript; } \\
\text { study concept or design; study } \\
\text { supervision; and obtaining funding }\end{array}$ \\
\hline $\begin{array}{l}\text { Ekaterina } \\
\text { Rogaeva, } \\
\text { PhD }\end{array}$ & $\begin{array}{l}\text { University of } \\
\text { Toronto, ON, } \\
\text { Canada }\end{array}$ & $\begin{array}{l}\text { Drafting/revising the manuscript and } \\
\text { acquisition of data }\end{array}$ \\
\hline $\begin{array}{l}\text { Gary W. } \\
\text { Beecham, } \\
\text { PhD }\end{array}$ & $\begin{array}{l}\text { University of } \\
\text { Miami, FL }\end{array}$ & $\begin{array}{l}\text { Drafting/revising the manuscript; } \\
\text { analysis or interpretation of data; } \\
\text { study supervision; and obtaining } \\
\text { funding }\end{array}$ \\
\hline
\end{tabular}

\section{References}

1. Alzheimer's Association. 2019 Alzheimer's disease facts and figures. Alzheimers Dement 2019;15:321-387.

2. Bateman RJ, Xiong C, Benzinger TL, et al. Clinical and biomarker changes in dominantly inherited Alzheimer's disease. N Engl J Med 2012;367:795-804.

3. Kukull WA, Higdon R, Bowen JD, et al. Dementia and Alzheimer disease incidence: a prospective cohort study. Arch Neurol 2002;59:1737-1746.

4. Wingo TS, Lah JJ, Levey AI, Cutler DJ. Autosomal recessive causes likely in earlyonset Alzheimer disease. Arch Neurol 2012;69:59-64.

5. Barber IS, Braae A, Clement N, et al. Mutation analysis of sporadic early-onset Alzheimer's disease using the NeuroX array. Neurobiol Aging 2017;49: 215.e211-215.e218.

6. Alzheimer's A. Early-Onset Dementia: A National Challenge, a Future Crisis. Washington, DC: Alzheimer's Association; 2006.

7. Lambert MA, Bickel H, Prince M, et al. Estimating the burden of early onset dementia; systematic review of disease prevalence. Eur J Neurol 2014;21:563-569.

8. Renvoize E, Hanson M, Dale M. Prevalence and causes of young onset dementia in an English health district. Int J Geriatr Psychiatry 2011;26:106-107.

9. Dubois B, Feldman HH, Jacova C, et al. Advancing research diagnostic criteria for Alzheimer's disease: the IWG-2 criteria. Lancet Neurol 2014;13:614-629. 
10. van der Flier WM, Pijnenburg YA, Fox NC, Scheltens P. Early-onset versus late-onset Alzheimer's disease: the case of the missing APOE varepsilon4 allele. Lancet Neurol 2011;10:280-288.

11. Bateman RJ, Aisen PS, De Strooper B, et al. Autosomal-dominant Alzheimer's disease a review and proposal for the prevention of Alzheimer's disease. Alzheimers Res Ther 2011;3:1.

12. Ryan NS, Rossor MN. Correlating familial Alzheimer's disease gene mutations with clinical phenotype. Biomark Med 2010;4:99-112.

13. Wattmo C, Wallin AK. Early- versus late-onset Alzheimer's disease in clinical practice cognitive and global outcomes over 3 years. Alzheimers Res Ther 2017;9:70.

14. Koedam EL, Pijnenburg YA, Deeg DJ, et al. Early-onset dementia is associated with higher mortality. Dement Geriatr Cogn Disord 2008;26:147-152.

15. Stanley K, Walker Z. Do patients with young onset Alzheimer's disease deteriorate faster than those with late onset Alzheimer's disease? A review of the literature. Int Psychogeriatr 2014;26:1945-1953.

16. Tang M, Ryman DC, McDade E, et al. Neurological manifestations of autosomal dominant familial Alzheimer's disease: a comparison of the published literature with the Dominantly Inherited Alzheimer Network observational study (DIAN-OBS). Lancet Neurol 2016;15:1317-1325.

17. McDade E, Wang G, Gordon BA, et al. Longitudinal cognitive and biomarker changes in dominantly inherited Alzheimer disease. Neurology 2018;91:e1295-e1306.

18. Ryan NS, Nicholas JM, Weston PSJ, et al. Clinical phenotype and genetic association in autosomal dominant familial Alzheimer's disease: a case series. Lancet Neurol 2016; 15:1326-1335.

19. Jonsson T, Atwal JK, Steinberg S, et al. A mutation in APP protects against Alzheimer's disease and age-related cognitive decline. Nature 2012;488:96-99.

20. Serrano-Pozo A, Frosch MP, Masliah E, Hyman BT. Neuropathological alterations in Alzheimer disease. Cold Spring Harb Perspect Med 2011;1:a006189.

21. Arai Y, Yamazaki M, Mori O, Muramatsu H, Asano G, Katayama Y. Alpha-synucleinpositive structures in cases with sporadic Alzheimer's disease: morphology and its relationship to tau aggregation. Brain Res 2001;888:287-296.

22. Twohig D, Rodriguez-Vieitez E, Sando SB, et al. The relevance of cerebrospinal fluid alpha-synuclein levels to sporadic and familial Alzheimer's disease. Acta Neuropathol Commun 2018;6:130.

23. Hyman BT, Phelps $\mathrm{CH}$, Beach TG, et al. National Institute on Aging-Alzheimer's Association guidelines for the neuropathologic assessment of Alzheimer's disease. Alzheimers Dement 2012;8:1-13.

24. Ingelsson M, Fukumoto $\mathrm{H}$, Newell $\mathrm{KL}$, et al. Early Abeta accumulation and progressive synaptic loss, gliosis, and tangle formation in $\mathrm{AD}$ brain. Neurology 2004;62: 925-931.

25. Savva GM, Wharton SB, Ince PG, et al. Age, neuropathology, and dementia. N Engl J Med 2009;360:2302-2309.

26. Middleton LE, Grinberg LT, Miller B, Kawas C, Yaffe K. Neuropathologic features associated with Alzheimer disease diagnosis: age matters. Neurology 2011;77:1737-1744.

27. Haroutunian V, Schnaider-Beeri M, Schmeidler J, et al. Role of the neuropathology of Alzheimer disease in dementia in the oldest-old. Arch Neurol 2008;65:1211-1217.

28. Marshall GA, Fairbanks LA, Tekin S, Vinters HV, Cummings JL. Early-onset Alzheimer's disease is associated with greater pathologic burden. J Geriatr Psychiatry Neurol 2007;20:29-33.

29. Scholl M, Ossenkoppele R, Strandberg O, et al. Distinct 18F-AV-1451 tau PET retention patterns in early- and late-onset Alzheimer's disease. Brain 2017;140:2286-2294.

30. Youn YC, Jang JW, Han SH, et al. (11)C-PIB PET imaging reveals that amyloid deposition in cases with early-onset Alzheimer's disease in the absence of known mutations retains higher levels of PIB in the basal ganglia. Clin Interv Aging 2017;12: 1041-1048.

31. Hirano $\mathrm{S}$, Shinotoh $\mathrm{H}$, Shimada $\mathrm{H}$, et al. Voxel-based acetylcholinesterase PET study in early and late onset Alzheimer's disease. J Alzheimers Dis 2018;62: 1539-1548.

32. Lowe VJ, Wiste HJ, Senjem ML, et al. Widespread brain tau and its association with ageing, Braak stage and Alzheimer's dementia. Brain 2018;141:271-287.

33. Crook R, Verkkoniemi A, Perez-Tur J, et al. A variant of Alzheimer's disease with spastic paraparesis and unusual plaques due to deletion of exon 9 of presenilin 1 . Nat Med 1998;4:452-455

34. Rogaeva E. The solved and unsolved mysteries of the genetics of early-onset Alz heimer's disease. Neuromolecular Med 2002;2:1-10.

35. Le TV, Crook R, Hardy J, Dickson DW. Cotton wool plaques in non-familial lateonset Alzheimer disease. J Neuropathol Exp Neurol 2001;60:1051-1061.

36. Qiang W, Yau WM, Lu JX, Collinge J, Tycko R. Structural variation in amyloid-beta fibrils from Alzheimer's disease clinical subtypes. Nature 2017;541:217-221.
37. Cairns NJ, Perrin RJ, Franklin EE, et al. Neuropathologic assessment of participants in two multi-center longitudinal observational studies: the Alzheimer Disease Neuroimaging Initiative (ADNI) and the Dominantly Inherited Alzheimer Network (DIAN). Neuropathology 2015;35:390-400.

38. Consensus recommendations for the postmortem diagnosis of Alzheimer's disease. The National Institute on Aging, and Reagan Institute Working Group on diagnostic criteria for the neuropathological assessment of Alzheimer's disease. Neurobiol Aging 1997; 18:S1-S2.

39. Mirra SS. The CERAD neuropathology protocol and consensus recommendations for the postmortem diagnosis of Alzheimer's disease: a commentary. Neurobiol Aging 1997;18:S91-S94.

40. Khachaturian ZS. Diagnosis of Alzheimer's disease. Arch Neurol 1985;42:1097-1105.

41. Benzinger TL, Blazey T, Jack CR Jr, et al. Regional variability of imaging biomarkers in autosomal dominant Alzheimer's disease. Proc Natl Acad Sci USA 2013;110 E4502-E4509.

42. Knight WD, Okello AA, Ryan NS, et al. Carbon-11-Pittsburgh compound B positron emission tomography imaging of amyloid deposition in presenilin 1 mutation carriers. Brain 2011;134:293-300

43. Gordon BA, Blazey TM, Christensen J, et al. Tau PET in autosomal dominant Alzheimer's disease: relationship with cognition, dementia and other biomarkers. Brain 2019;142:1063-1076.

44. Suarez-Calvet M, Capell A, Araque Caballero MA, et al. CSF progranulin increases in the course of Alzheimer's disease and is associated with sTREM2, neurodegeneration and cognitive decline. EMBO Mol Med 2018;10:e9712.

45. Moller C, Vrenken H, Jiskoot L, et al. Different patterns of gray matter atrophy in early- and late-onset Alzheimer's disease. Neurobiol Aging 2013;34:2014-2022.

46. Rabinovici GD, Furst AJ, Alkalay A, et al. Increased metabolic vulnerability in earlyonset Alzheimer's disease is not related to amyloid burden. Brain 2010;133:512-528.

47. Cho H, Choi JY, Lee SH, et al. Excessive tau accumulation in the parieto-occipita cortex characterizes early-onset Alzheimer's disease. Neurobiol Aging 2017;53: 103-111.

48. Schöll M, Ossenkoppele R, Strandberg O, et al. Distinct 18F-AV-1451 tau PET retention patterns in early- and late-onset Alzheimer's disease. Brain 2017;140 2286-2294.

49. Holland D, Desikan RS, Dale AM, McEvoy LK; Alzheimer's Disease Neuroimaging Initiative. Rates of decline in Alzheimer disease decrease with age. PLoS One 2012;7: e42325.

50. Shi L, Baird AL, Westwood S, et al. A decade of blood biomarkers for Alzheimer's disease research: an evolving field, improving study designs, and the challenge of replication. J Alzheimers Dis 2018;62:1181-1198.

51. Hampel H, Vergallo A, Afshar M, et al. Blood-based systems biology biomarkers for next-generation clinical trials in Alzheimer's disease. Dialogues Clin Neurosci 2019; 21:177-191.

52. Ovod V, Ramsey KN, Mawuenyega KG, et al. Amyloid beta concentrations and stable isotope labeling kinetics of human plasma specific to central nervous system amyloidosis. Alzheimers Dement 2017;13:841-849.

53. Nabers A, Perna L, Lange J, et al. Amyloid blood biomarker detects Alzheimer's disease. EMBO Mol Med 2018;10:e8763.

54. Palmqvist S, Janelidze S, Stomrud E, et al. Performance of fully automated plasma assays as screening tests for Alzheimer disease-related beta-amyloid status. JAMA Neurol 2019;76:1060-1069.

55. Kaneko N, Nakamura A, Washimi Y, et al. Novel plasma biomarker surrogatin cerebral amyloid deposition. Proc Jpn Acad Ser B Phys Biol Sci 2014;90:353-364.

56. Janelidze S, Stomrud E, Palmqvist S, et al. Plasma beta-amyloid in Alzheimer's disease and vascular disease. Sci Rep 2016;6:26801.

57. Mattsson N, Andreasson U, Zetterberg H, Blennow K; Alzheimer's Disease Neuroimaging Initiative. Association of plasma neurofilament light with neurodegeneration in patients with Alzheimer disease. JAMA Neurol 2017;74:557-566.

58. Weston PSJ, Poole T, Ryan NS, et al. Serum neurofilament light in familial Alzheimer disease: a marker of early neurodegeneration. Neurology 2017;89:2167-2175.

59. Preische O, Schultz SA, Apel A, et al. Serum neurofilament dynamics predicts neurodegeneration and clinical progression in presymptomatic Alzheimer's disease. Nat Med 2019;25:277-283.

60. Bridel C, van Wieringen WN, Zetterberg $\mathrm{H}$, et al. Diagnostic value of cerebrospinal fluid neurofilament light protein in neurology: a systematic review and meta-analysis. JAMA Neurol 2019;76:1035-1048.

Additional references (e-1 to e-78) to this article are listed at links.lww.com/NXG/ A320 


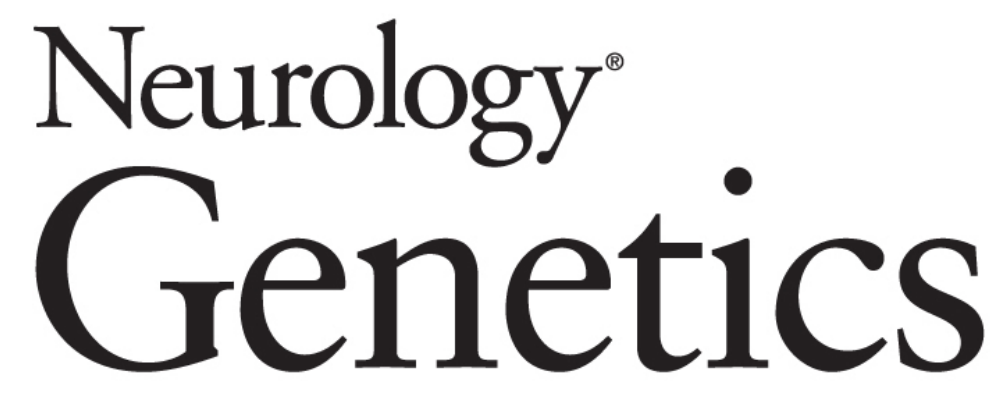

\section{Late-onset vs nonmendelian early-onset Alzheimer disease: A distinction without a difference? \\ Christiane Reitz, Ekaterina Rogaeva and Gary W. Beecham \\ Neurol Genet 2020;6; \\ DOI 10.1212/NXG.0000000000000512}

This information is current as of October 6, 2020

\begin{abstract}
Updated Information \& Services

References

Citations

Permissions \& Licensing

Reprints

including high resolution figures, can be found at: http://ng.neurology.org/content/6/5/e512.full.html

This article cites 59 articles, 4 of which you can access for free at: http://ng.neurology.org/content/6/5/e512.full.html\#\#ref-list-1

This article has been cited by 5 HighWire-hosted articles: http://ng.neurology.org/content/6/5/e512.full.html\#\#otherarticles its entirety can be found online at:

http://ng.neurology.org/misc/about.xhtml\#permissions

Information about ordering reprints can be found online: http://ng.neurology.org/misc/addir.xhtml\#reprintsus
\end{abstract}

Information about reproducing this article in parts (figures,tables) or in

Neurol Genet is an official journal of the American Academy of Neurology. Published since April 2015, it is an open-access, online-only, continuous publication journal. Copyright Copyright ( 2020 The Author(s). Published by Wolters Kluwer Health, Inc. on behalf of the American Academy of Neurology.. All rights reserved. Online ISSN: 2376-7839.

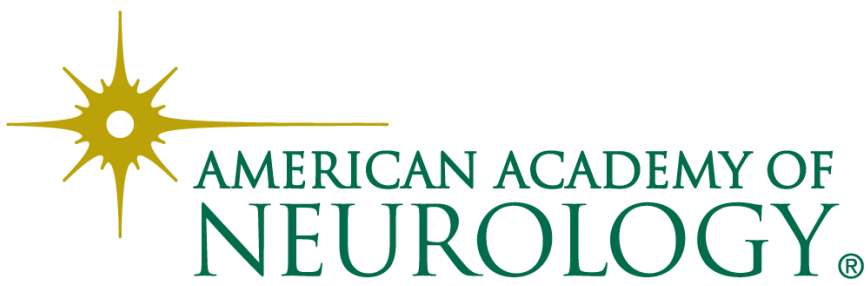

